\title{
Identificación de las características esenciales para aplicaciones Android de e-health orientadas al monitoreo de la obesidad
}

\author{
Carlos Alberto Veloz Vidal ${ }^{1}$, Viviana Bustos Amador ${ }^{1}$, Francisco Álvarez Rodríguez ${ }^{1}$, \\ Ricardo Mendoza González ${ }^{2,3}$ \\ ${ }^{1}$ Universidad Autónoma de Aguascalientes, Centro de Ciencias Básicas, Dpto. Sistemas de \\ Información Av. Universidad \#940, Cd. Universitaria, CP. 20131, Aguascalientes, México. \\ ${ }^{2}$ Universidad Politécnica de Aguascalientes, Dirección de Posgrado e Investigación, Calle Paseo \\ San Gerardo No. 207. Fracc. San Gerardo. C.P. 20342. Aguascalientes, México \\ ${ }^{3}$ Instituto Tecnológico de Aguascalientes, Departamento de Sistemas y Computación, Av. Adolfo \\ López Mateos 1801, Ote. Fracc. Bonagens. C.P. 20257. Aguascalientes, México \\ \{carlosa.veloz, ic.viviana.b\}@gmail.com, fjalvar@correo.uaa.mx, \\ ricardo.mendoza@upa.edu.mx,mendozagric@mail.ita.mx
}

\begin{abstract}
Resumen. Actualmente México ocupa el primer lugar mundial en obesidad, lo que representa uno de los principales problemas de salud pública del país. Esto ha despertado el interés, y la necesidad de que la comunidad tecnológica y científica se unan a los especialistas de la salud, para generar estrategias que refuercen los tratamientos preventivos tradicionales para el control del peso. Una de estas estrategias es el desarrollo de aplicaciones e-health para el sistema operativo Android. Si bien, existen aplicaciones disponibles para este fin, la mayoría no cuentan con un respaldo de profesionales de la salud, haciendo de sus diagnósticos, y/o sugerencias poco confiables. Para contribuir en la reducción de este problema, se analizaron las aplicaciones Android de obesidad más populares, esto, permitió establecer características clave a considerar en aplicaciones de e-health. Dichos hallazgos se materializaron en una aplicación para el Monitoreo de la Obesidad, donde los resultados obtenidos se discuten al final del artículo.
\end{abstract}

Palabras clave: obesidad, dispositivos móviles, Android, e-health, cuestionario de hábitos alimenticios. 


\section{Introducción}

Actualmente el $30 \%$ de la población adulta en México tiene problemas de obesidad a diferencia del $28 \%$ alcanzado por Estados Unidos, esto de acuerdo con datos presentados en [1]. Con respecto a la población infantil, México ocupa el cuarto lugar de prevalencia mundial de obesidad, aproximadamente $28.1 \%$ en niños y $29 \%$ en niñas, superado solo por Grecia, Estados Unidos e Italia [2].

La obesidad y el sobrepeso se consideran enfermedades no transmisibles (ENT), las cuales tienen repercusiones en los gastos de salud, tanto de los gobiernos, como de los individuos que las padecen. Las consecuencias de las ENT impactan directamente en la productividad laboral, desempeño escolar, calidad de vida y bienestar tanto físico como emocional del individuo.

En 2008, el 80\% del total de las defunciones (29 millones) por ENT se produjeron en países de ingresos bajos y medios. La obesidad tiene diferentes orígenes como lo son la predisposición genética, estilo de vida, falta de actividad física, el insuficiente consumo de frutas y verduras, la cultura, la educación, la globalización, el entorno político y social, por mencionar algunos de los principales factores. Básicamente el problema de obesidad y sobrepeso se resumen en un desequilibrio energético entre las calorías que se consumen y las que se gastan. En todo el mundo se ha incrementado la ingesta de alimentos hipercalóricos (ricos en grasa, sal y azucares pero pobres en vitaminas, minerales y micronutrientes) y se ha reducido la actividad física (como resultado de un estilo de vida más sedentaria, ya sea por el trabajo, cambio en los medios de transporte y la creciente urbanización) [3, 4].

Por otro lado, el uso de los dispositivos móviles (smartphones y tablets) se ha incrementado a tal grado que se han convertido en una alternativa al cómputo tradicional (laptops y PCs de escritorio), haciendo más simple la movilidad, usabilidad y portabilidad de estos dentro de la población en general, lo cual los convierte en una valiosa plataforma para el desarrollo de aplicaciones relacionadas con temas de salud.

Los dispositivos móviles son una vía particularmente atractiva para el desarrollo de aplicaciones de la salud, debido a: (1) la adopción generalizada de dispositivos móviles con capacidades técnicas cada vez más potentes [5], (2) la tendencia de las personas a llevar estos dispositivos con ellos a todas partes, (3) el apego de las personas a sus teléfonos [6], y (4) las características de sensibilización de contexto habilitadas a través de sensores y la información personal vía telefónica.

La dependencia de las personas a llevar siempre consigo un dispositivo móvil, además de la facilidad para desarrollar aplicaciones de e-health para el sistema operativo Android, permiten a las instituciones de salud así como a los desarrolladores independientes por igual, crear productos de gran aceptación entre la población que ataquen los padecimientos ya mencionados a través de diferentes frentes, como lo son aplicaciones que fomenten la actividad física, el cambio a una dieta más saludable, detección del estado de salud del usuario, monitoreo y seguimiento de enfermedades, etc. Sin embargo, 
Identificación de las características esenciales para aplicaciones android de e-health orientadas al ...

es importante que estas aplicaciones sean desarrolladas con apego a un estudio científico realizado por especialistas en el área de la salud, ya que al tratar situaciones muy delicadas del estilo de vida de los usuarios, se requiere que el diagnostico o recomendaciones sean las adecuadas, para beneficiar y mejorar la calidad de vida de los usuarios que las utilizan.

Lo anterior representa la principal motivación para la realización de este trabajo, cuyas secciones se distribuyen de la siguiente manera: la sección 2, presenta la problemática actual en las aplicaciones de e-health, seguida del estudio comparativo en la sección 3. El prototipo generado a partir de las características identificadas se describe en la sección 4, los resultados parciales de la aplicación desarrollada para el monitoreo de obesidad (disponible en [7]) se presentan en la sección 5. Finalmente las conclusiones, así como el camino a seguir en el corto plazo se presentan en la sección 6.

\section{Problemática}

Existen miles de aplicaciones disponibles en la Google Play Store listas para ser descargadas por los usuarios, sin embargo menos del $1 \%$ del total de aplicaciones disponibles se encuentran dentro de la categoría de Medicina. Al realizar una búsqueda en Google Play con las palabras: "control de peso", "obesidad test" y "obesidad diagnostico", se observa en los resultados que son muy pocas las aplicaciones que son desarrolladas (o por lo menos respaldadas) por instituciones o especialistas, ya que la gran mayoría de aplicaciones disponibles son creadas por desarrolladores independientes.

Esta situación representa un problema importante debido a que los resultados, diagnósticos, sugerencias/consejos, dietas, y demás resultados generados por las aplicaciones son poco confiables. Esto a la larga podría verse reflejado directamente en los volúmenes de descarga de las aplicaciones disponibles, al ser percibidas como poco confiables por los usuarios desde la lectura de la descripción en los repositorios como Google Play Store. Otro aspecto que puede percibirse como un punto de mejora, es sin duda que la mayoría de las aplicaciones relacionadas con obesidad o sobrepeso, se enfocan en presentar al usuario una serie de consejos o dietas que con el tiempo le permitan llegar a su peso "ideal", el cual en muchas aplicaciones es establecido por él mismo usuario pudiendo desencadenar un problema de salud adicional. Un número menor de aplicaciones se orientan al seguimiento del peso del usuario a través de la captura periódica de su peso, y otra información indicando si se está o no progresando en el logro de las metas establecidas. Al igual que en el caso anterior, dichas metas son precisadas por el mismo usuario o por valores proporcionados por la aplicación, basados en tablas que no indican sus orígenes lo cual nuevamente representa un riesgo para la salud, generando resultados contraproducentes.

Con el fin de contribuir en la reducción de esta brecha se pretende identificar las características principales de una aplicación para el monitoreo de la obesidad a partir de 
un estudio comparativo entre las aplicaciones (orientadas a este fin) más populares en Google Play Store. Las características encontradas ayudarán a determinar que se puede mejorar conformando un conjunto de elementos esenciales los cuales podrán materializarse finalmente en un prototipo. Ambas contribuciones se describen en las siguientes secciones.

\section{Estudio comparativo}

Con el fin de establecer el punto de partida de esta investigación, se realizó un estudio comparativo en el que se consideraron las 5 aplicaciones mejor valoradas, de acuerdo a las estadísticas mostradas por el repositorio Google Play Store, tras realizar la búsqueda interna "control de peso". Cabe mencionar que la búsqueda generó un total de 240 aplicaciones encontradas aproximadamente.

Las aplicaciones seleccionadas para el estudio fueron: 1) Monitorea tu peso (https://play.google.com/store/apps/details?id=monitoryourweight.bustan.net); 2) Control de peso (https://play.google.com/store/apps/details?id=org.angel.weightcontrol); 3) Perder peso, adelgazar, IMC (https://play.google.com/store/apps/details?id=com.cryofy.android. weightmeterfree); 4) Peso asistente (https://play.google.com/store/apps/details?id=com. ikdong.weight); 5) Peso diary (https://play.google.com/store/apps/details?id= weight.manager). Llama la atención que ninguna de estas aplicaciones fue desarrollada (o está respaldada) por un hospital, institución o especialista. Otro aspecto importante de las aplicaciones analizadas, es que prácticamente ninguna fundamenta sus resultados/diagnósticos presentados al usuario. Adicionalmente, las aplicaciones seleccionadas reflejaban claramente (en diferentes proporciones) los aspectos problemáticos descritos en la sección 2 de este documento. Relacionado con esto, los resultados mostrados en cada una de las aplicaciones analizadas dejan la conclusión e interpretación de dicha información a criterio del usuario lo cual puede generar confusiones y malas interpretaciones desembocando muy probablemente en problemas de salud mayores.

A continuación se describen las características principales que fueron encontradas en las aplicaciones analizadas. Solo dos de ellas no tienen soporte multiusuario (Peso asistente y Peso diary), sin embargo, esta funcionalidad puede descartarse en el desarrollo de aplicaciones de e-health, ya que los dispositivos móviles por lo general son dispositivos personales. La mayoría de las aplicaciones seleccionadas, permiten el cálculo del IMC (Índice de Masa Corporal) del usuario y cálculo del porcentaje de grasa corporal, cuentan también con una opción que permite guardar el resultado o diagnostico obtenido para su posterior consulta. Varias de estas aplicaciones muestran al usuario consejos o dietas para alcanzar un peso "ideal" definido por él, estas aplicaciones son Monitorea tu peso, Control de peso y Perder peso, adelgazar, IMC; por lo que incluyen funciones que invitan a los usuarios a capturar su peso de manera periódica (diaria o semanal), además del uso de gráficas para mostrar el "progreso" obtenido para alcanzar el peso deseado. 
Varias de estas aplicaciones incluyen publicidad dentro de las interfaces de usuario, lo cual aparte de distraer al usuario del objetivo principal de la aplicación, también impacta en el número de descargas total, donde la aplicación que cuenta con el mayor número es Perder peso, que se encuentra en el rango de 1,000,000 a 5,000,000 millones de descargas totales, seguida de Monitorea tu peso, que se encuentra dentro del rango de 500,000 a 1,000,00 descargas, las aplicaciones con el menor número son Peso asistente y Peso diary, lo cual las sitúa en el rango de 100,000 a 500,000 descargas.

El análisis realizado, permitió identificar varios aspectos de mejora que pueden integrarse en las siguientes características básicas para una aplicación de e-health para obesidad (y que sin duda podrías ser válidas para otras áreas de salud) son: 1) Tamaño relativamente pequeño, no debe pesar demasiados $\mathrm{Mb}$ ya que no resultará atractivo para un usuario descargar una aplicación "pesada", 2) Exenta de publicidad, las aplicaciones de e-health no deben contener publicidad, ni cualquier otro distractor que desvíe la atención del objetivo original de la aplicación, 3) Respaldo científico, al tratar temas delicados de salud, debe de haber un estudio científico por profesionales en el área, que permita generar un diagnóstico adecuado a la condición de cada usuario; esto se debe indicar en la ficha de Google Play, 4) Ser gratuita, ya que el objetivo principal de este tipo de aplicaciones es mejorar la calidad de vida de los usuarios que las utilizan, finalmente 5) Debe contener solo los elementos necesarios, es decir, debe ser una interfaz limpia que emplea solo los elementos necesarios para su funcionamiento.

\section{$4 \quad$ Prototipo generado a partir de las características identificadas}

Las características arrojadas por el análisis fueron integradas en un prototipo de una aplicación "Monitoreo de Obesidad" la cual se dispuso en Google Play Store (https://play.google.com/store/apps/details?id=com.mipaquete.prototipo1\&hl=es). El prototipo se compone de un módulo para la aplicación del cuestionario de hábitos alimenticios, otro para el cálculo del IMC-Estos cálculos se basan en la Norma Oficial Mexicana NOM043-SSA2-2012 [7]. Cabe mencionar que esta norma fue elaborada por distintas unidades administrativas e instituciones del Sector Salud Mexicano con el fin de establecer las pautas hacia una alimentación saludable y adecuada con estricto apego a los criterios de la OMS (Organización Mundial de la Salud) válidos para la población mundial - . La información obtenida en estos módulos, es la base del diagnóstico que se le proporciona al usuario, el cual posteriormente se almacena en una base de datos local y finalmente se muestran una serie de (semi-personalizados) consejos con base al diagnóstico generado que lo orientan hacia un estilo de vida más saludable.

El cuestionario utilizado consta de 36 preguntas que permiten conocer los hábitos alimentarios del paciente, dichas preguntas están clasificadas en tres grupos que son: 1) Hábitos alimenticios, 2) Actividad física y 3) Estado de ánimo. Un aspecto considerado para la elección de este cuestionario es, que a través de las preguntas realizadas se obtiene 
información sobre el consumo de azúcar en la dieta, incluso sobre el tipo de alimentos que frecuentemente consumen los usuarios con obesidad. Además, el cuestionario empleado, permite identificar de manera más precisa el estado de salud de un usuario ya que considera factores como el consumo de azúcar, alimentación saludable, ejercicio físico, contenido calórico, bienestar psicológico, tipo de alimentos, conocimiento y control y por ultimo consumo de alcohol [8]. Se optó por elegir este cuestionario, ya que es fácil de implementar dentro de una aplicación para dispositivos móviles, permite establecer una valoración inicial sobre el estado de salud del usuario, la cual complementa los tratamientos preventivos tradicionales para el cuidado de la obesidad y el sobrepeso.

Al implementar el cuestionario dentro de una aplicación en Android, se realizó teniendo en cuenta las características propuestas para el desarrollo de aplicaciones de ehealth, por lo que la interfaz de usuario incluye imágenes alusivas al tema de salud, se muestra la pregunta y las opciones disponibles para su respuesta, en la figura 1, se puede apreciar un prototipo de la pantalla a utilizar.

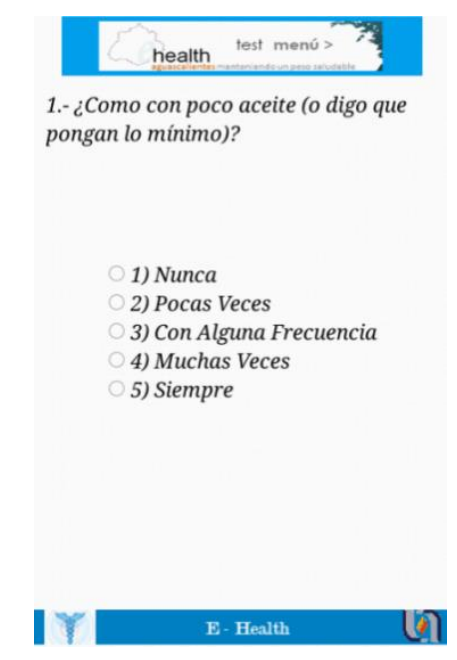

Fig. 1. Pantalla de las interfaz de Monitoreo de Obesidad.

Las interfaces del cuestionario, contienen solo los elementos necesarios para que el usuario responda con tranquilidad cada uno de las preguntas que se le presentan, sin publicidad de ningún tipo que lo distraiga del objetivo original de esta aplicación, que en este caso es el diagnostico preventivo. Una vez que ha concluido el cuestionario se pide al usuario que ingrese su altura y su peso, para poder calcular su IMC. Como se mencionó anteriormente la información de estos dos módulos, constituye la base del resultado que se muestra al usuario. En caso de que el usuario cuente con algún diagnostico anterior, este se obtiene de la base de datos local, y se compara con el diagnostico actual, esto se realiza 
Identificación de las características esenciales para aplicaciones android de e-health orientadas al ...

para mostrar si ha habido alguna mejoría en los hábitos alimenticios del usuario, en la figura 2 se muestra el prototipo de la pantalla de resultados.

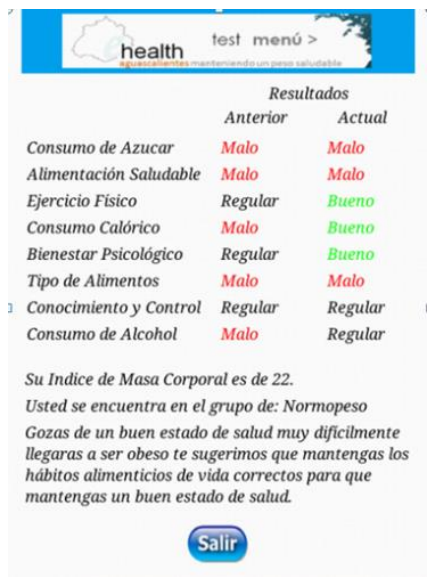

Fig. 2. Diagnóstico del estado de salud.

El diagnostico que se muestra en la figura anterior se conforma de los resultados del último diagnostico disponible y el actual, donde para cada uno de los factores de riesgo anteriormente mencionados se acompañan de una leyenda "Bueno", "Regular" o "Malo", la cual se obtiene de comparar el valor obtenido para cada uno de ellos contra el valor ideal como se menciona en [8]. Posteriormente se presenta en la siguiente línea el valor del IMC del usuario, indicando además el grupo donde se encuentra y un mensaje semipersonalizado de acuerdo a esta valoración inicial.

Con los resultados obtenidos, se determinan los consejos que se mostraran al usuario, enfocándose primero en combatir aquellos factores de riesgo con la leyenda "Malo", ya que son los que tienen mayor prioridad, y a los cuales el usuario debería prestar mayor cuidado; los factores de riesgo con la leyenda "Regular" son consejos que invitan al usuario a cambiar la ingesta de alimentos fritos, por alimentos a la plancha, por mencionar un ejemplo, pues son cambios menores los que debe realizar el usuario en su dieta para atender los factores de riesgo correspondientes. Por último, los consejos que se muestran para los factores de riesgo con la leyenda "Bueno", buscan fortalecer esos hábitos donde el usuario no tiene aparentemente ningún problema por lo detectado en la valoración inicial, dichos consejos son de carácter informativo y de acuerdo a lo recomendado por la Norma Oficial Mexicana NOM-043-SSA2-2012.

Es importante mencionar, que tanto la valoración inicial como los consejos de salud que se muestran al usuario, en ningún momento pueden reemplazar el diagnostico medico realizado por alguna institución de salud o un especialista; por el contrario, a través de su 
uso en dispositivos móviles se busca que sirvan para complementar el tratamiento tradicional contra la obesidad y el sobrepeso.

\section{$5 \quad$ Resultados}

Los datos que se presentan en esta sección, fueron obtenidos a través de la consola para desarrolladores de Google, la cual permite dar un seguimiento adecuado sobre la aceptación y distribución de la aplicación, permite conocer las instalaciones actuales, instalaciones en total desde que la aplicación fue publicada, valoración media, comentarios de los usuarios, etc.

Como se mencionó anteriormente, la aplicación Monitoreo de Obesidad se distribuye gratuitamente en todos los países donde Google Play tiene presencia, como se puede apreciar en la figura 3, lo cual ha permitido que esta aplicación sea probado por usuarios de México, España, Argentina, Chile, Colombia, etc.

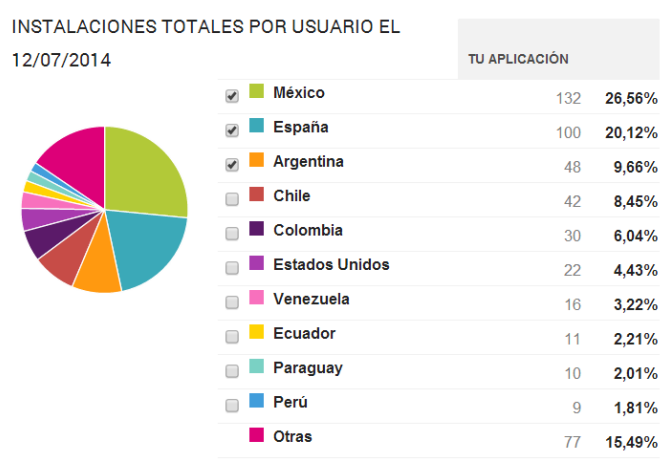

Fig. 3. Países donde se ha descargado la aplicación Monitoreo de Obesidad.

La aplicación Monitoreo de Obesidad, fue publicada por primera vez en Octubre de 2013, y a la fecha se han liberado dos versiones la aplicación para reparar errores menores en el funcionamiento y la interfaz de usuario, siendo la versión actual Monitoreo de Obesidad 1.2, publicada el 9 de Febrero de 2014. A principios del mes de Julio del año en curso se cuentan con las siguientes estadísticas: la aplicación ha sido descargada un total de 492 ocasiones y se encuentra activa en 61 dispositivos; cuenta con una valoración de 4.17 estrellas de un máximo de 5.

Existen aplicaciones disponibles que son de gran aceptación entre los usuarios, lo cual las posiciona entre los primeros lugares de descarga, sin embargo, la mayoría de estas aplicaciones pueden mejorarse con las características mencionadas anteriormente, para construir aplicaciones de familia, donde la información, diagnósticos y/o consejos que se 
Identificación de las características esenciales para aplicaciones android de e-health orientadas al ...

muestran al usuario sean respaldados por instituciones de salud o especialistas y aun así sean de utilidad para el tratamiento y diagnóstico de distintos padecimientos.

\section{Conclusiones y trabajo futuro}

Durante este trabajo de investigación, se identificaron las características básicas que pudieran considerarse para el desarrollo de aplicaciones de e-health para obesidad, además se mencionó la importancia de que los resultados, diagnósticos y/o consejos que se muestran al usuario, deben contar con el respaldo de una institución de salud o bien por especialistas en el área, ya que al tratar temas relacionados con la salud, se debe tener cuidado con el tratamiento y la forma en que se hace llegar la información al usuario.

Los resultados obtenidos hasta el momento para la aplicación Monitoreo de Obesidad (ver en https://play.google.com/store/apps/details?id=com.mipaquete.prototipo1), utilizando las características propuestas para el desarrollo de aplicaciones de e-health, hacen que esta aplicación se encuentre catalogada en el rango de 100 a 500 descargas dentro de Google Play, es importante mencionar que la aplicación se distribuye gratuitamente en todos los países donde tiene alcance Google Play, lo que ha permitido que se tenga una calificación aceptable de 4.17 estrellas de un máximo de 5 , sin errores o fallos reportados hasta el momento esto de acuerdo a lo reportado por la consola de desarrolladores de Google.

Si bien, los resultados actuales son aceptables, se pretende realizar varias mejoras a la aplicación para aumentar el número de descargas y situarla dentro de las aplicaciones mejor valoradas y descargadas para el tratamiento de la obesidad. Entre las mejores a implementar en el corto plazo son mostrar consejos de salud de acuerdo a lo mencionado en la sección 4 de este trabajo de investigación, con lo que se espera que la aplicación actual sea de utilidad para el usuario, le permita dar un seguimiento real sobre su estado de salud y se convierta así, en una herramienta de apoyo entre especialistas para los tratamientos tradicionales de la obesidad y el sobrepeso.

\section{Referencias}

[1] Franco S.: Obesity and the Economics of Prevention: Fit not Fat. Organization for the Economic Cooperationand Development (OECD publishing); (2010)

[2] Franco S.: Obesity Update 2012. Organization for the Economic Cooperation and Development (OECD publishing); (2012)

[3] Plan de Acción Mundial para la Prevención y Control de las Enfermedades no Transmisibles 2013-2020, OMS. Disponible en: http://who.int/cardiovascular_diseases/15032013_updates_revised_draft_action_plan_spa nish.pdf 
[4] OMS (2006) Organización Mundial de la Salud. Obesidad y sobrepeso. Nota descriptiva no. 311 [en línea]. Disponible en http://www.who.int/mediacentre/factsheets/fs311/es/index.html, (2012)

[5] Pew Internet \& American Life Project. Internet, broadband, and cell phone statistics; Recuperado de: http://www.pewinternet.org/Reports/2010/Internet-broadband-and-cellphone-statistics.aspx? $\mathrm{r}=1,(2010)$

[6] Ventä L, Isomursu M, Ahtinen A, Ramiah S.: My Phone is a part of my soul - how people bond with their mobile phones. In: Proc UbiComm '08. IEEE Computer Society; p. 3117, (2008)

[7] NOM-043-SSA2-2012, Servicios básicos de salud. Promoción y educación de salud en materia alimentaria. Criterios para brindar orientación. (FUENTE: Diario Oficial de la federación de fecha 28 de Mayo de 2012)

[8] Castro Rodríguez P, Bellido Guerrero D, Pertega Díaz S.: Elaboración y validación de un nuevo cuestionario de hábitos alimentarios para pacientes con sobrepeso y obesidad. Endocrinol Nutr 2010; 57 (4): 130-139, (2010) 